\title{
Spot Speed Survey \& Analysis - A Case Study on Jalandhar-Ludhiana Road, NationalHighway-1, India
}

\author{
Bhargav Naidu.M \\ School of Civil Enginering, Lovely Professional University \\ Phagwara, Punjab - 144401, India \\ Email: bhargav.naidu818@gmail.com
}

\begin{abstract}
The Present Vehicle Spot Speed Study concentrates on measuring the speed characteristics at predetermined area under the natural conditions.The present spot speed studies are coordinated to assess the movement of rates of vehicles in a surge of activity at a particular region on a roadway. The proposed look into work goes from breaking down the modular speed of vehicles, highway outline component ,upper and lower speed restrain for regulation, planning and analysis, traffic operation, control and direction. The range, time and conditions of the study may be coordinated by its objective and expansion. In case approach paces to a merging are required, the estimations should be taken upstream of the intersection point going before vehicle deceleration for a possible stop at the intersection point. If the study requires free-stream speeds, the estimations should be taken in the midst of off-zenith times. A comparable method of reasoning should be taken after for estimations required in the midst of night conditions, wet black-top, and so forth. There are two ordinarily utilized ways to deal with gather vehicle speeds at spot areas: singular vehicle choice strategy and all-inspecting vehicle technique. The individual vehicle assurance system includes using a manual speed estimation method and is generally used for transient speed estimations. This segment focuses on the individual vehicle assurance methodology. In this present survey the average speed, variance \& standard deviation, median speed, modal speed \& percentile speed of various category of vehicles are determined and analyzed with the accident data of the area thereby finding the reason for the occurrence of accidents and determining the safe speed limits in the area and also providing measures for the improvement of road safety measures on national highway-1 like deciding existing movement operations and assessment of activity control gadgets, setting up roadway plan components, and measuring adequacy of activity control gadgets or movement programs, including signs and markings, activity operational changes, and speed implementation programs.
\end{abstract}

Keywords: Spot Speed, Modular Speed, Percentile Speed, Median Speed, Transient Speed.

\section{INTRODUCTION}

Speed is an essential measure to assess security of the street organize. Speed is likewise a critical transportation parameter in light of the fact that other than wellbeing, it identifies with time, solace, comfort, and financial aspects. As indicated by Currin (2001), rapid conveys high hazard, while low speed is moderately protected. The plan speed ought to be made relying upon the sheltered speed point of confinement of the street ${ }^{(1)}$ Speed is one of the activity operational components that ought to be considered in the outline of the street geometrics.

\section{A. Scope}

Spot speed studies are coordinated to assess the movement of rates of vehicles in a surge of activity at a particular region on a roadway. ${ }^{(2)}$ The proposed look into work goes for breaking down the modular speed of vehicles, highway outline component, upper and lower speed restrain for regulation, planning and analysis, traffic operation -control and direction. ${ }^{(3)}$

\section{B. Objective}

Figure out if complaints about speeding in the study location are valid and to assess the impacts of present physical enhancements present on the location and then build up passing and non-passing zones based on the speed study and then correlating data obtained from speed study with the accident data of the particular location and then finally preparing the draft for the planning of geometric alignment and also building the speed zone for the safety of road users based on the spot speed data. 


\section{METHODOLOGY}

A comparable method of reasoning should be taken after for estimations required in the midst of night conditions, wet black-top, and so forth. There are two ordinarily utilized ways to deal with gather vehicle speeds at spot areas: singular vehicle choice strategy and all-inspecting vehicle technique. The individual vehicle assurance system includes using a manual speed estimation method and is generally used for transient speed estimations. ${ }^{(4)}$ The all-looking at methodology uses automated as a piece of road or roadside estimation equipment (e.g., pneumatic tubes, standard acknowledgment circles, point circles, et cetera.) And is fitting to use for structure execution checking system. This segment focuses on the individual vehicle assurance methodology.

The data is collected in view of arbitrarily inspecting singular vehicle speed over short time period. It depends on upon watching the time required by vehicle to cover a short separation of Roadway. ${ }^{(3)}$ Mid-Block of the road andstraight and level sections of highways are selected to calculate the spot speed of the vehicles whereas junctions, crossing points are avoided as accurate determination of spot speeds cannot be done. The survey is done during peak hours of a day during weekdays where a regular traffic flow is present and external influences like traffic signals and busy access roads are avoided as vehicles tend to reduce their speeds at these points hence giving raise to inaccuracy. ${ }^{(6)}$

\section{AREA UNDER SURVEY}

The study region is extended from NH1 and village road in Chaheru to that of Ludhiana. Chaheru is a town in the locale of Kapurthala, Punjab, India. Closest urban regions are Phagwara and Jalandhar. According to the 2011 measurements, by the Indian Government, Chaheru has 509 families, and a population of 2,458 out of which 1248 are men.

Ludhiana is a metropolitan city in Punjab, and is the biggest city north of Delhi. It is the biggest city in the state, with an expected populace of 1,693,653 as of the 2011 Evaluation. The populace increments significantly amid the collecting season because of the relocation of workers from the eastern conditions of Uttar Pradesh, Bihar, Odisha and Delhi. Ludhiana is found 98 kilometers (61 miles) west of the state capital Chandigarh on NH 95 and is halfway situated on National Expressway 1, which keeps running from the Indian capital New 51\%), A few noteworthy National Roadways, NH1, NH95, NH11, NH20 go through the city. The transportation administrations are given by state possessed Punjab Roadways and private transport administrators.

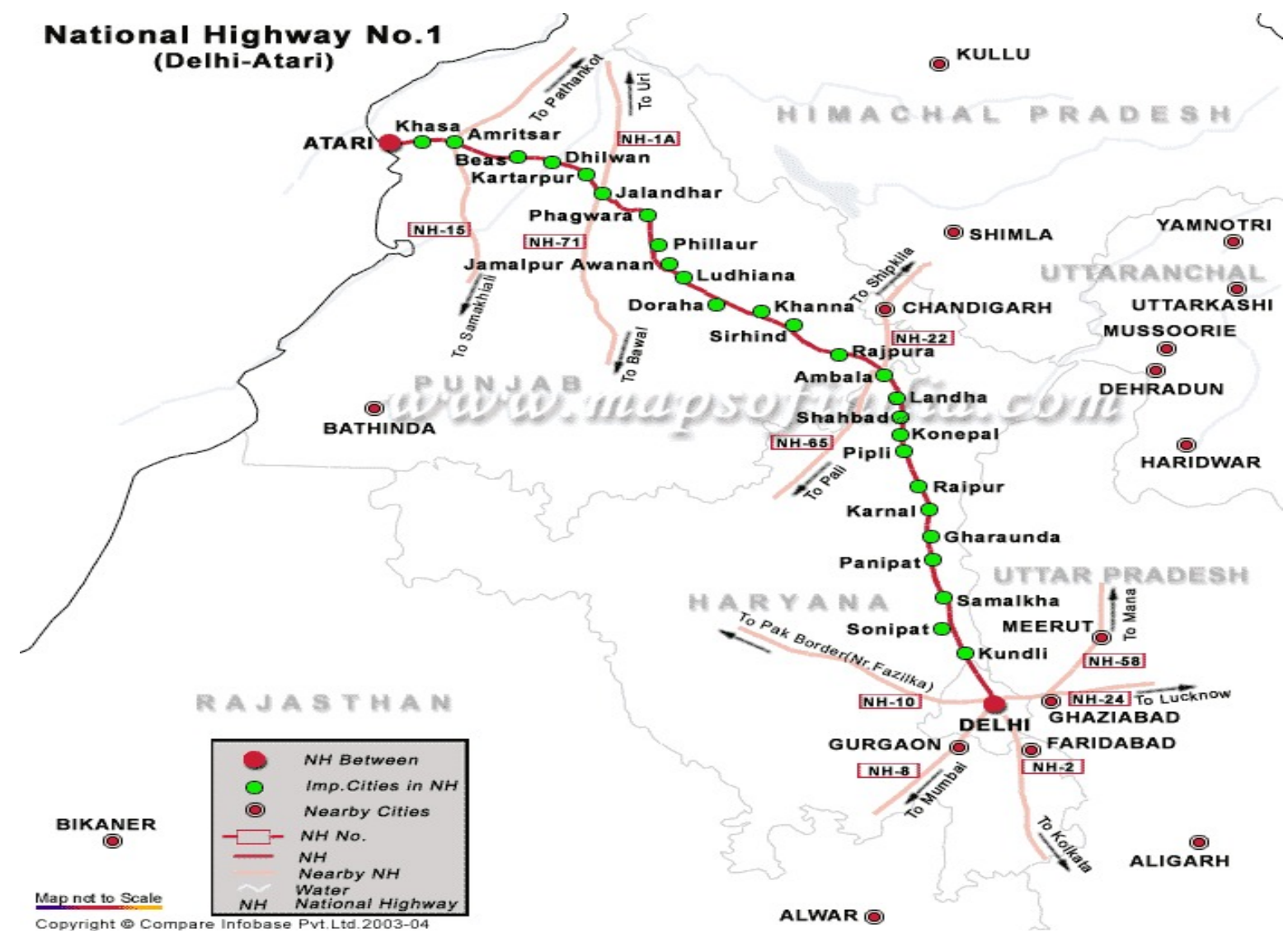

Fig. 1 National Highway No. 1 (Delhi-Atari) 


\section{DATA ANALYSIS}

The collected data is processed by a few elucidating measurements to get a few thoughts regarding the appropriation of the speed information. (Take note of that numerous measurable investigations utilized as a part of activity building whether the accepted information is regularly appropriated. $\rightarrow$ In this way, the objective is to check whether they are truly regularly appropriate.

A. Typical Descriptive Statistics:

1. Average speed

2. Variance and standard deviation

3. Median speed

4. Modal speed (or Modal speed range $\rightarrow$ Needs a histogram)

5. The $i^{\text {th }}$-percentile spot speed

B. Analysis of Spot Speed data:

1. To prepare frequency distribution curve.

2. Select number of classes typically between $8-20$ (another framework: choose stretch out for a class size of 8 , then choose keep running for a class size of 20 , by detaching the refinement among maximum and minimum speeds by 8 then by 20 , then selecting a range between these greatest and min ranges).

3. The mid value for each class is used as a speed value for that class frequency distribution curve (speed mid qualities versus frequency distribution curve).

4. Cumulative distribution (maximum breaking points of speed classes versus frequency distribution curve)

\section{RESULTS \& DISCUSSIONS}

\begin{tabular}{|ll}
\hline LOCATION: CHAHERU & DATE: $2 / 10 / 16$ \\
WEATHER: SUNNY DAY & TIME: 10 A.M. TO 2P.M. \\
TYPE OF ROAD : NH1 & BASE LENGTH: 50m \\
MEASUREMENT TECHNIQUE: MANUALLY & VEHICLE: BUSES(50)
\end{tabular}

TABLE 1. Frequency Distribution of Buses

\begin{tabular}{|c|c|c|c|c|c|}
\hline $\begin{array}{c}\text { SPEED } \\
\text { RANGE Kmph }\end{array}$ & $\begin{array}{l}\text { MID } \\
\text { SPEED (x) }\end{array}$ & $\begin{array}{l}\text { FREQUENCY } \\
\text { (f) }\end{array}$ & $\begin{array}{l}\text { \%FREQUENCY } \\
\text { (\%f) }\end{array}$ & $\begin{array}{l}\text { \%CUMMULATIVE } \\
\text { FREQUENCY (\%c.f) }\end{array}$ & (f $\left.\mathbf{f}_{\mathbf{x}}\right)$ \\
\hline $40-50$ & 45 & 0 & 0 & 0 & 0 \\
\hline $50-60$ & 55 & 3 & 6 & 48 & 165 \\
\hline $60-70$ & 65 & 21 & 42 & 82 & 1365 \\
\hline $70-80$ & 75 & 17 & 34 & 98 & 680 \\
\hline $80-90$ & 85 & 8 & 16 & 100 & 95 \\
\hline $90-100$ & 95 & 1 & 2 & 100 & 0 \\
\hline $100-110$ & 105 & 0 & 0 & & $\sum \mathrm{fx}=3580$ \\
\hline & & $\sum \mathrm{f}=50$ & & & \\
\hline
\end{tabular}

AVERAGE SPEED FOR BUSES $=\sum f x / \sum f=(3580 / 50)=71.6 \mathrm{kmph}$ 


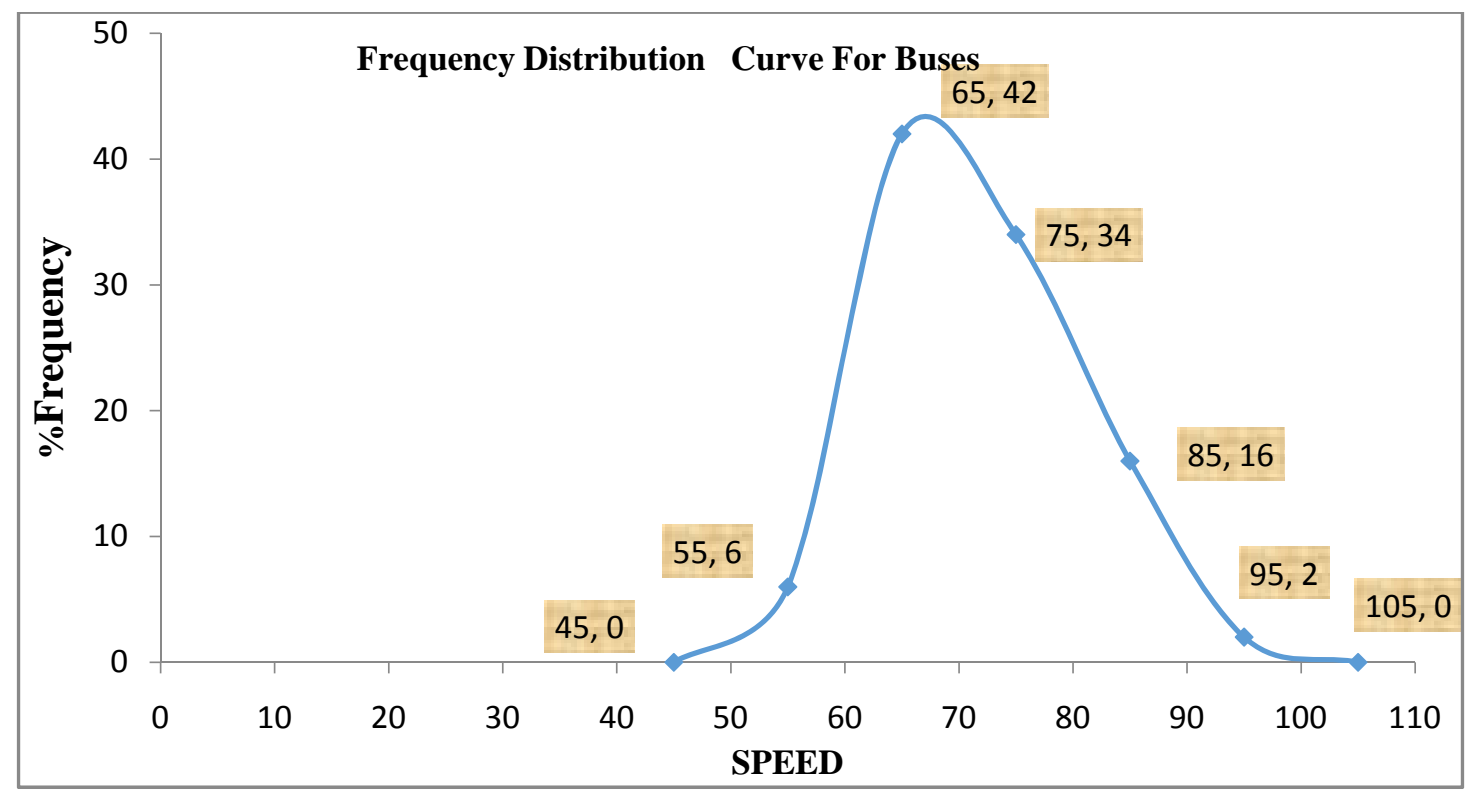

Fig. 2 Frequency Distribution Curve for Buses

From frequencyDistribution curveModal speed $=65 \mathrm{kmph}$

Therefore the most preferred speed at which maximumProportion of buses travel is the modal speed $=65 \mathrm{kmph}$

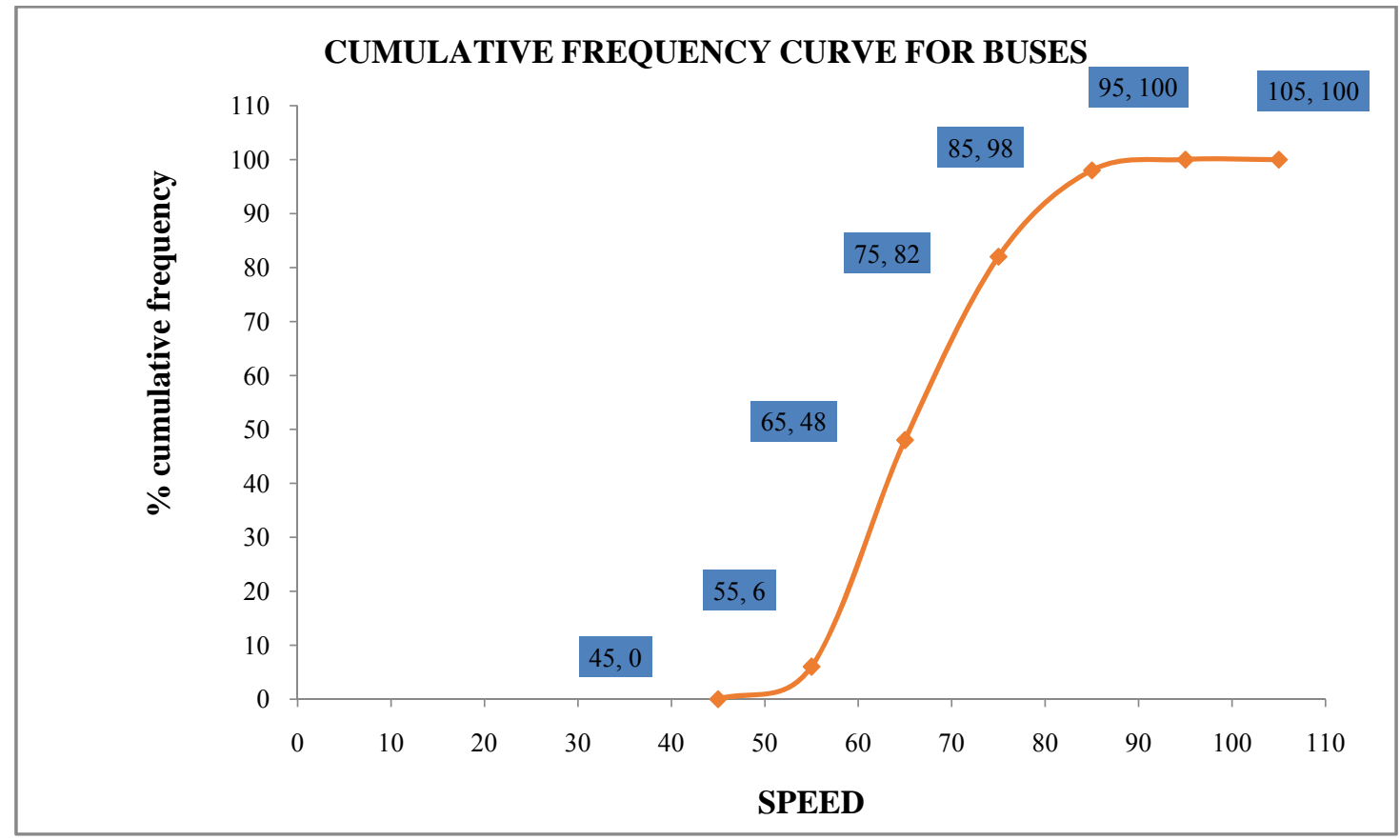

Fig. 3 Cumulative Frequency Curve for buses

For NH1 Chaheru for Buses

1. Upper Speed

2. Lower Speed

3. Design Speed

4. Median Speed $85^{\text {th }}$ Percentile Speed $=80 \mathrm{Kmph}$

$=15^{\text {th }}$ PercentileSpeed $=60 \mathrm{Kmph}$

$=\quad 98^{\text {th }}$ Percentile Speed $=85 \mathrm{Kmph}$

$=50^{\text {th }}$ Percentile Speed $=70 \mathrm{Kmph}$ 
TABLE 2. Data Analysis for buses

\begin{tabular}{|c|c|c|c|c|}
\hline $\begin{array}{c}\text { SPEED RANGE } \\
\text { Kmph }\end{array}$ & MID SPEED (v) & (q) & (q.v.) & (q/v) \\
\hline $40-50$ & 45 & 0 & 0 & 0 \\
\hline $50-60$ & 55 & 3 & 165 & 0.0545 \\
\hline $60-70$ & 65 & 21 & 1365 & 0.3230 \\
\hline $70-80$ & 75 & 17 & 1275 & 0.2266 \\
\hline $80-90$ & 85 & 8 & 680 & 0.0941 \\
\hline $90-100$ & 95 & 1 & 95 & 0.0105 \\
\hline $100-110$ & 105 & 0 & 0 & 0.0 \\
\hline & & $\sum \mathrm{f}=50$ & $\sum \mathrm{qv}=3580$ & $\sum \mathrm{q} / \mathrm{v}=0.7087$ \\
\hline
\end{tabular}

Space mean speed (Vs.)

$=\sum \frac{q}{v} / \sum f=(0.7087) /(50)=0.01417 \mathrm{kmph}$

Time mean speed $(\mathrm{Vt})$

$=\sum q v / \sum f=(3580) / 50=71.6 \mathrm{kmph}$

Standard deviation $(\mathrm{S} 2)=$ Vs. $(\mathrm{Vt}-\mathrm{Vs})=0.01417(71.6-0.01417)=1.014$

TABLE 3. Frequency Distribution of Cars

\begin{tabular}{|c|c|c|c|c|c|}
\hline \multicolumn{4}{|c|}{$\begin{array}{l}\text { LOCATION: CHAHERU } \\
\text { WEATHER: SUNNY DAY } \\
\text { TYPE OF ROAD : NH1 } \\
\text { MEASUREMENT TECHNIQUE: MANUALLY }\end{array}$} & \multicolumn{2}{|c|}{$\begin{array}{l}\text { DATE: 2/10/16 } \\
\text { TIME: } 10 \text { A.M. TO 2P.M. } \\
\text { BASE LENGTH: } 50 \mathrm{~m} \\
\text { VEHICLE: } \operatorname{cars(50)}\end{array}$} \\
\hline $\begin{array}{c}\text { SPEED } \\
\text { RANGE Kmph }\end{array}$ & $\begin{array}{c}\text { MID } \\
\text { SPEED (x) }\end{array}$ & $\begin{array}{l}\text { FREQUENCY } \\
\text { (f) }\end{array}$ & $\begin{array}{l}\text { \%FREQUENCY } \\
\text { (\%f) }\end{array}$ & $\begin{array}{l}\text { \%CUMMULATIV } \\
\text { E FREQUENCY } \\
(\% \mathrm{c} . \mathrm{f})\end{array}$ & (fx) \\
\hline $30-40$ & 35 & 0 & 0 & 0 & 0 \\
\hline $40-50$ & 45 & 2 & 4 & 4 & 90 \\
\hline $50-60$ & 55 & 6 & 12 & 16 & 330 \\
\hline $60-70$ & 65 & 11 & 22 & 38 & 715 \\
\hline $70-80$ & 75 & 13 & 26 & 64 & 975 \\
\hline $80-90$ & 85 & 11 & 22 & 86 & 935 \\
\hline $90-100$ & 95 & 3 & 6 & 92 & 285 \\
\hline $100-110$ & 105 & 2 & 4 & 96 & 210 \\
\hline $110-120$ & 115 & 2 & 4 & 100 & 230 \\
\hline & & $\sum \mathrm{f}=50$ & & & $\sum \mathrm{fx}=3770$ \\
\hline
\end{tabular}

AVERAGE SPEED FOR CARS $=\sum f x / \sum f=(3770 / 50)=75.4 \mathrm{kmph}$ 


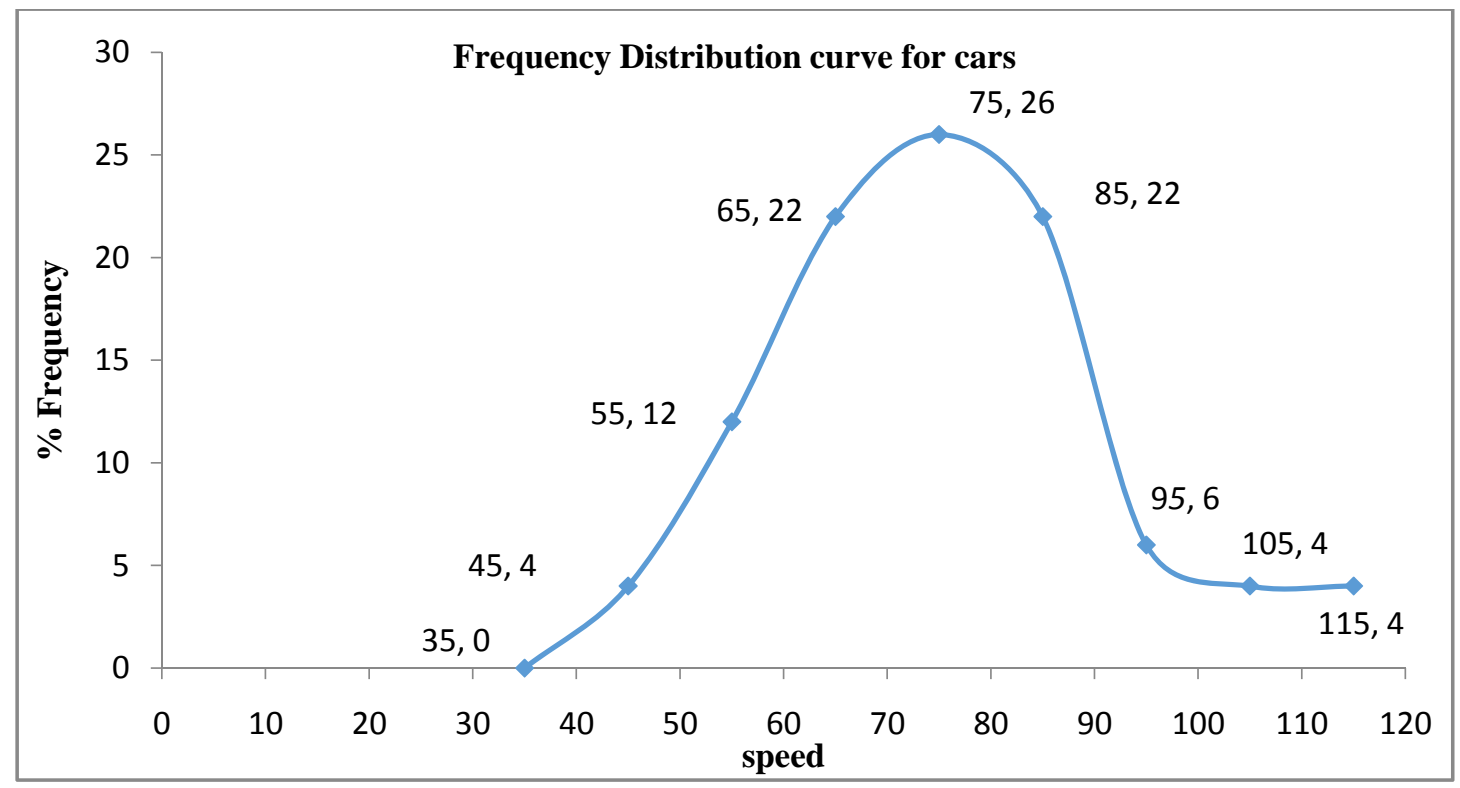

Fig. 4 Frequency Distribution Curve for Cars

From frequencyDistribution curveModal speed $=75 \mathrm{kmph}$

Therefore the most preferred speed at which maximumProportion of cars travel is the MODAL SPEED = $75 \mathrm{KMPH}$

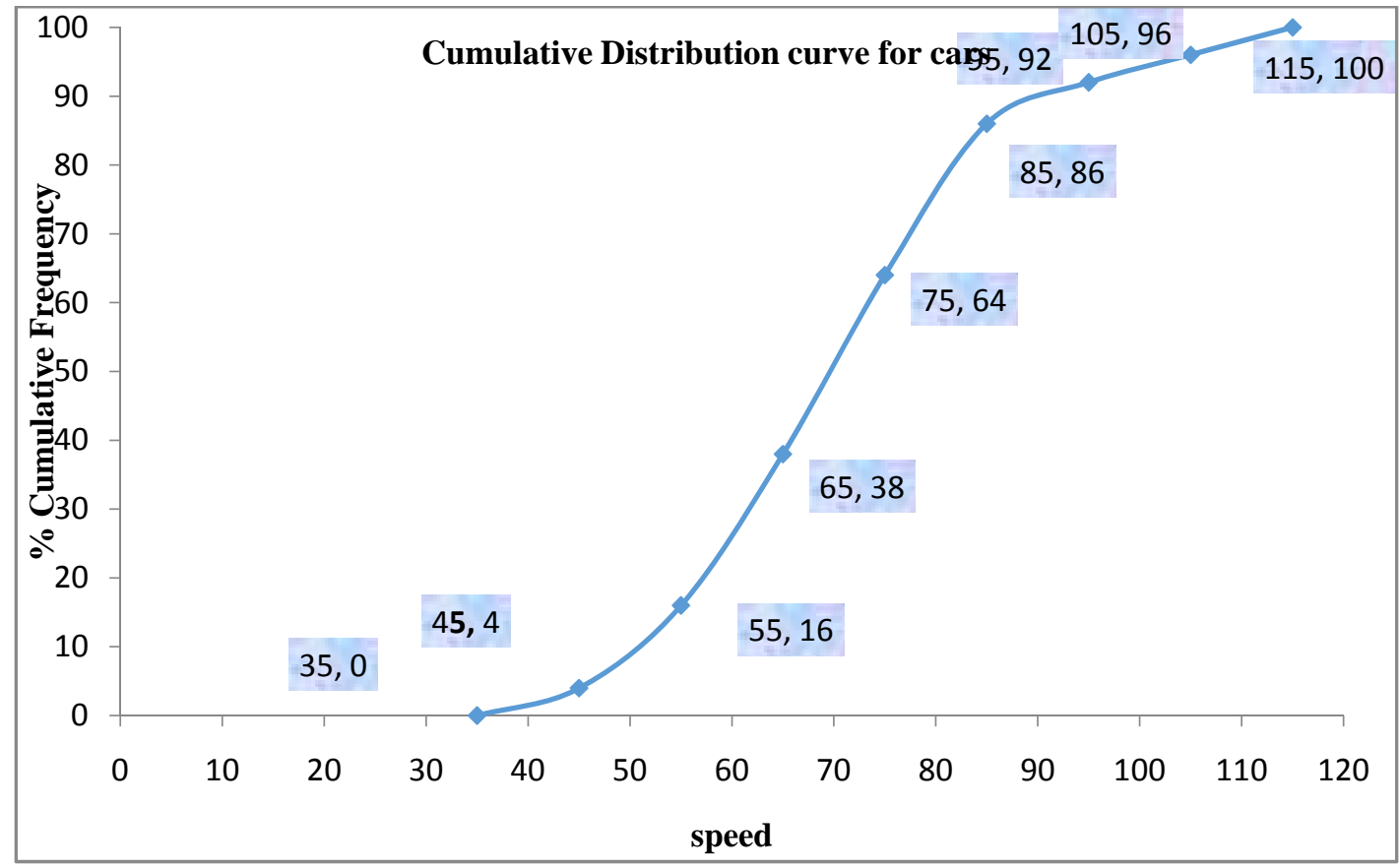

Fig. 5 Cumulative Distribution Curve for Cars

\section{FOR NH1 CHAHERU FOR BUSES}

1. UPPER SPEED

2. LOWER SPEED

3. DESIGN SPEED

4. MEDIAN SPEED $=\quad 85^{\text {th }}$ percentile speed $=80 \mathrm{kmph}$

$=\quad 15^{\text {th }}$ percentilespeed $=60 \mathrm{kmph}$

$98^{\text {th }}$ percentile speed $=85 \mathrm{kmph}$

$=50^{\text {th }}$ percentile speed $=70 \mathrm{kmph}$ 
TABLE 4. Data Analysis for Cars

\begin{tabular}{|c|c|c|c|c|}
\hline SPEED RANGE Kmph & MID SPEED (v) & (q) & (q.v.) & (q/v) \\
\hline $30-40$ & 35 & 0 & 0 & 0 \\
\hline $40-50$ & 45 & 2 & 90 & 0.0444 \\
\hline $50-60$ & 55 & 6 & 330 & 0.1090 \\
\hline $60-70$ & 65 & 11 & 715 & 0.1692 \\
\hline $70-80$ & 75 & 13 & 975 & 0.1733 \\
\hline $80-90$ & 85 & 11 & 935 & 0.1294 \\
\hline $90-100$ & 95 & 3 & 285 & 0.0315 \\
\hline $100-110$ & 105 & 2 & 210 & 0.0190 \\
\hline $110-120$ & 115 & 2 & 230 & 0.0173 \\
\hline & & $\sum \mathrm{f}=50$ & $\sum \mathrm{qv}=3770$ & $\sum \mathrm{q} / \mathrm{v}=0.6931$ \\
\hline
\end{tabular}

Space mean speed (Vs)

$=\sum \frac{q}{v} / \sum f=(0.6931) /(50)=0.0138 \mathrm{kmph}$

Time mean speed $(\mathrm{Vt})$

$=\sum q v / \sum f=(3370) / 50=75.4 \mathrm{kmph}$

Standard deviation (S2)

$=\mathrm{Vs} .(\mathrm{Vt}-\mathrm{Vs})=0.0138(75.4-0.0138)=1.04$

TABLE 5. Frequency Distribution of Trucks

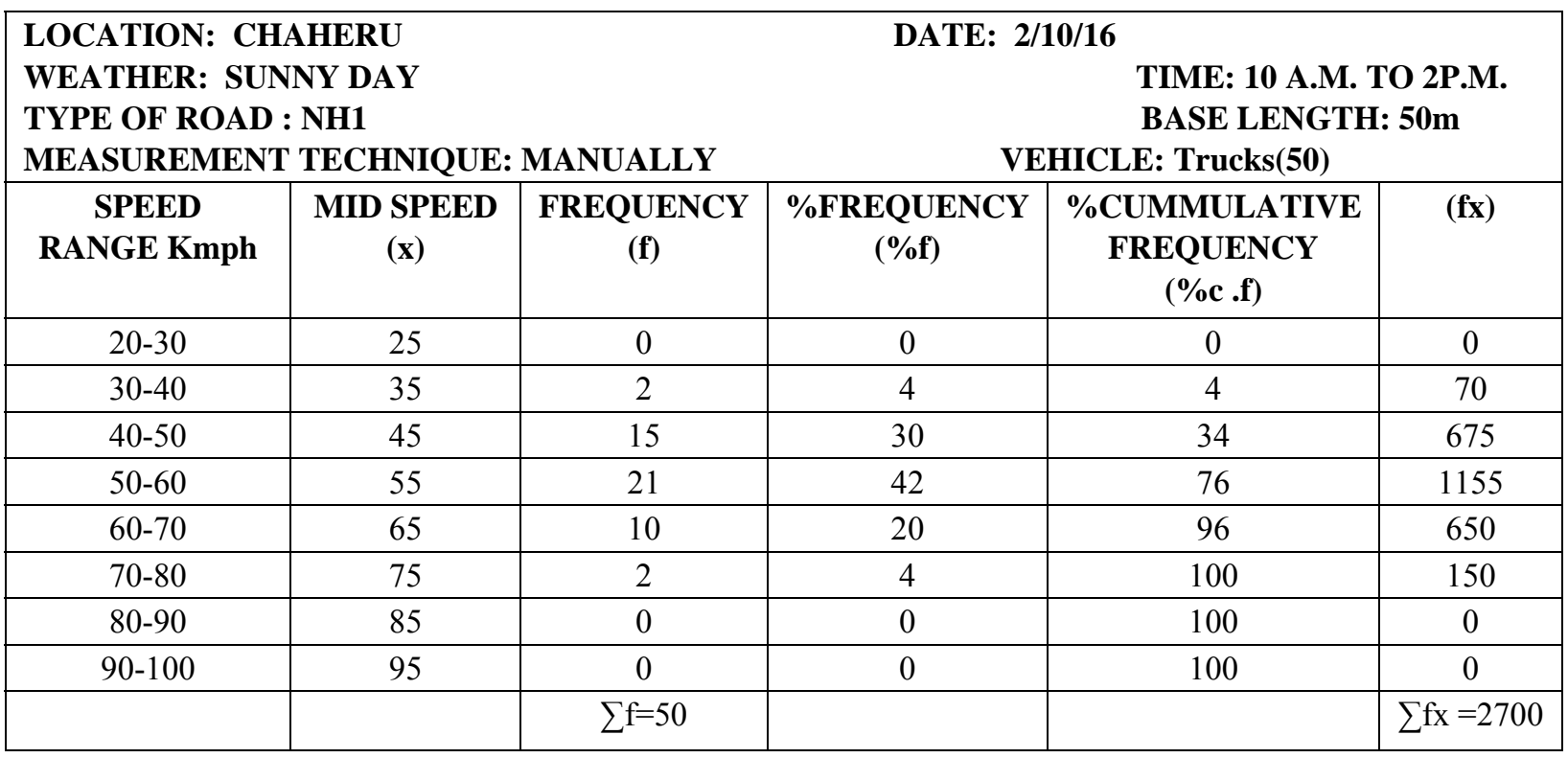

AVERAGE SPEED FOR CARS $=\sum f x / \sum f=(2700 / 50)=54 \mathrm{kmph}$ 


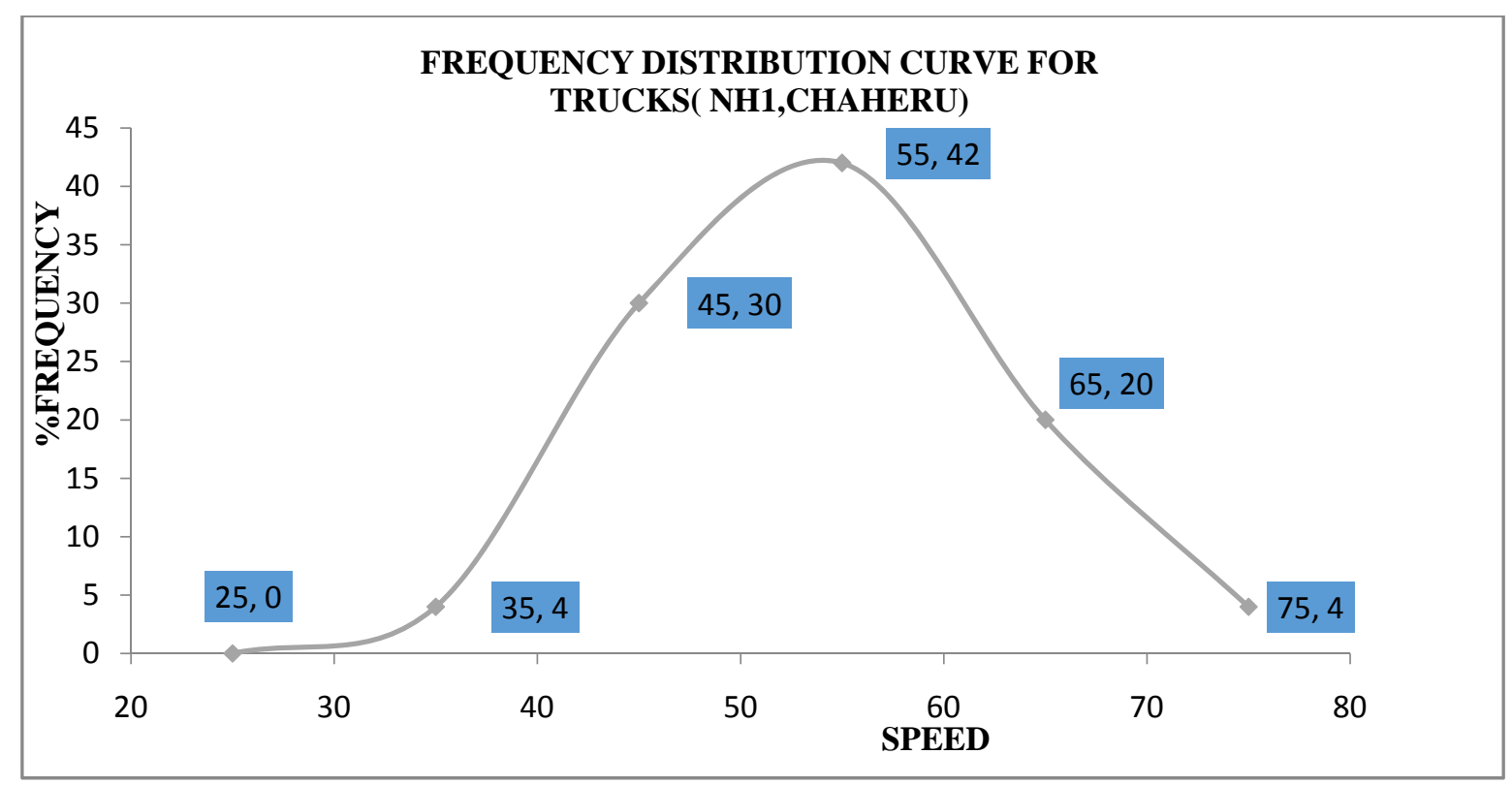

Fig. 6 Frequency Distribution Curve of Trucks

From frequencyDistribution curveModal speed $=55 \mathrm{kmph}$

Therefore the most preferred speed at which maximumProportion of trucks travel is the MODAL SPEED $=55$ $\mathrm{KMPH}$

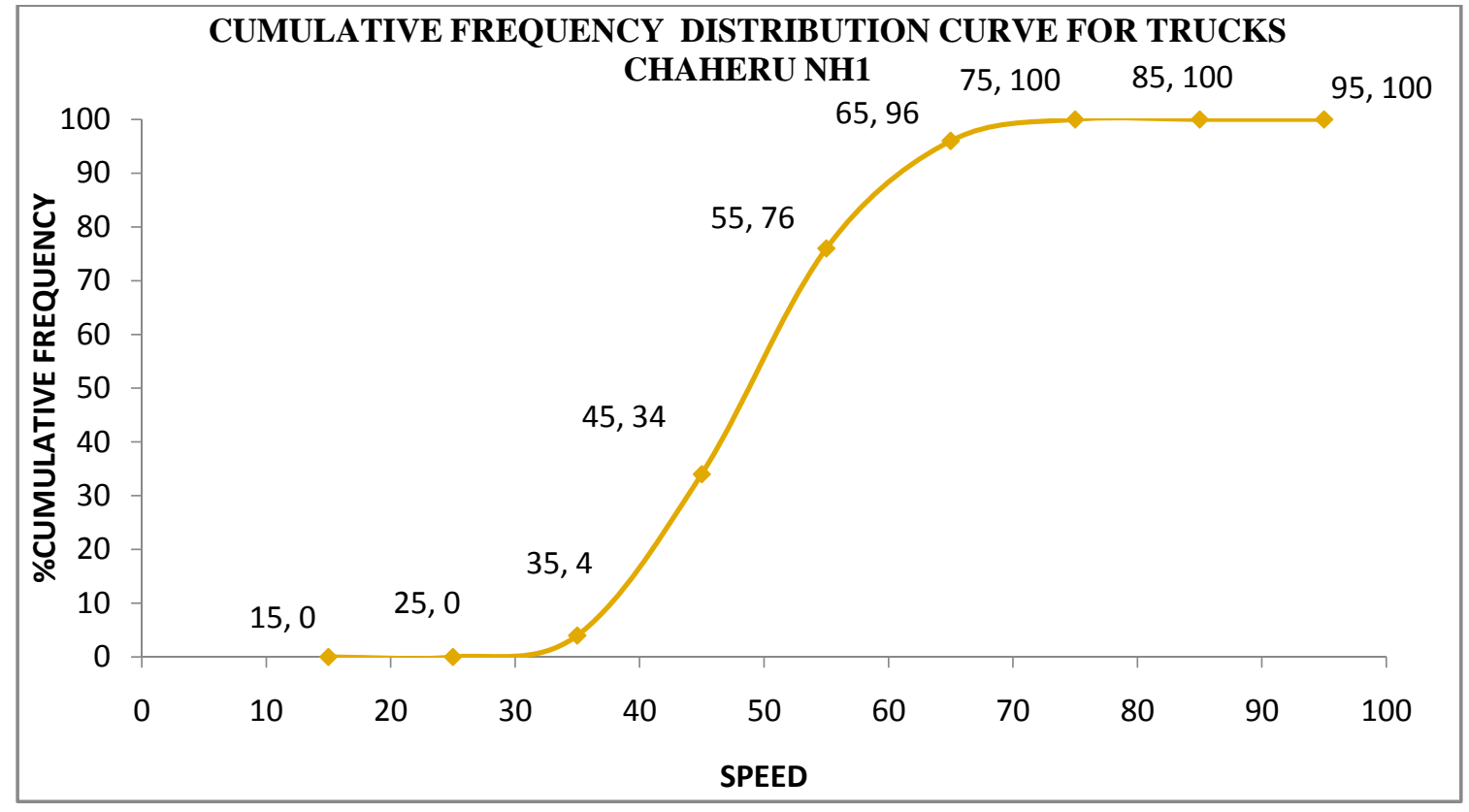

Fig. 7 Cumulative Frequency Distribution Curve of Trucks

1. UPPER SPEED $=85^{\text {th }}$ percentile speed $=60 \mathrm{kmph}$

2. LOWER SPEED $=15^{\text {th }}$ percentile speed $=40 \mathrm{kmph}$

3. DESIGN SPEED $=98^{\text {th }}$ percentile speed $=70 \mathrm{kmph}$

4. MEDIAN SPEED $=50^{\text {th }}$ percentile speed $=50 \mathrm{kmph}$ 
TABLE 6. Data Analysis of Trucks

\begin{tabular}{|c|c|c|c|c|}
\hline SPEED RANGE Kmph & MID SPEED (v) & (q) & (q.v.) & (q/v) \\
\hline $20-30$ & 25 & 0 & 0 & 0 \\
\hline $30-40$ & 35 & 2 & 70 & 0.057 \\
\hline $40-50$ & 45 & 15 & 675 & 0.333 \\
\hline $50-60$ & 55 & 21 & 1155 & 0.381 \\
\hline $60-70$ & 65 & 10 & 650 & 0.153 \\
\hline $70-80$ & 75 & 2 & 150 & 0.026 \\
\hline $80-90$ & 85 & 0 & 0 & 0.000 \\
\hline & & $\sum \mathrm{f}=50$ & $\sum \mathrm{qv}=2700$ & $\sum \mathrm{q} / \mathrm{v}=0.95$ \\
\hline
\end{tabular}

Space mean speed (Vs)

$=\sum \frac{q}{v} / \sum f=(0.95) /(50)=0.019 \mathrm{kmph}$

Time mean speed $(\mathrm{Vt})$

$=\sum q v / \sum f=(2700) / 50=54 \mathrm{kmph}$

Standard deviation (S2)

$=\mathrm{Vs}(\mathrm{Vt}-\mathrm{Vs})=0.019(54-0.019)=1.025$

TABLE 7. Frequency Distribution of Two Wheelers

\begin{tabular}{|c|c|c|c|c|c|}
\hline \multicolumn{4}{|c|}{$\begin{array}{l}\text { LOCATION: CHAHERU } \\
\text { WEATHER: SUNNY DAY } \\
\text { TYPE OF ROAD : NH1 } \\
\text { MEASUREMENT TECHNIQUE: MANUALLY }\end{array}$} & \multicolumn{2}{|c|}{$\begin{array}{l}\text { ATE: } 2 / 10 / 16 \\
\text { TIME: } 10 \text { A.M. TO 2P.M. } \\
\text { BASE LENGTH: 50m } \\
\text { VEHICLE: Two wheelers (50) }\end{array}$} \\
\hline $\begin{array}{c}\text { SPEED } \\
\text { RANGE } \\
\text { Kmph }\end{array}$ & $\begin{array}{c}\text { MID } \\
\text { SPEED } \\
\text { (x) }\end{array}$ & $\begin{array}{l}\text { FREQUENCY } \\
\text { (f) }\end{array}$ & $\begin{array}{c}\text { \%FREQUENCY } \\
(\% f)\end{array}$ & $\begin{array}{c}\text { \%CUMMULATIVE } \\
\text { FREQUENCY } \\
\text { (\%c.f) }\end{array}$ & $(f x)$ \\
\hline $30-40$ & 35 & 0 & 0 & 0 & 0 \\
\hline $40-50$ & 45 & 1 & 2 & 2 & 45 \\
\hline $50-60$ & 55 & 12 & 24 & 26 & 660 \\
\hline $60-70$ & 65 & 16 & 32 & 58 & 1040 \\
\hline $70-80$ & 75 & 16 & 32 & 90 & 1200 \\
\hline $80-90$ & 85 & 4 & 8 & 98 & 340 \\
\hline \multirow[t]{2}{*}{$90-100$} & 95 & 1 & 2 & 100 & 95 \\
\hline & & $\sum \mathrm{f}=50$ & & & $\sum \mathrm{fx}=3380$ \\
\hline
\end{tabular}

AVERAGE SPEED FOR TWO WHEELER $=\sum f x / \sum f=(3380 / 50)=67.6 \mathrm{kmph}$ 


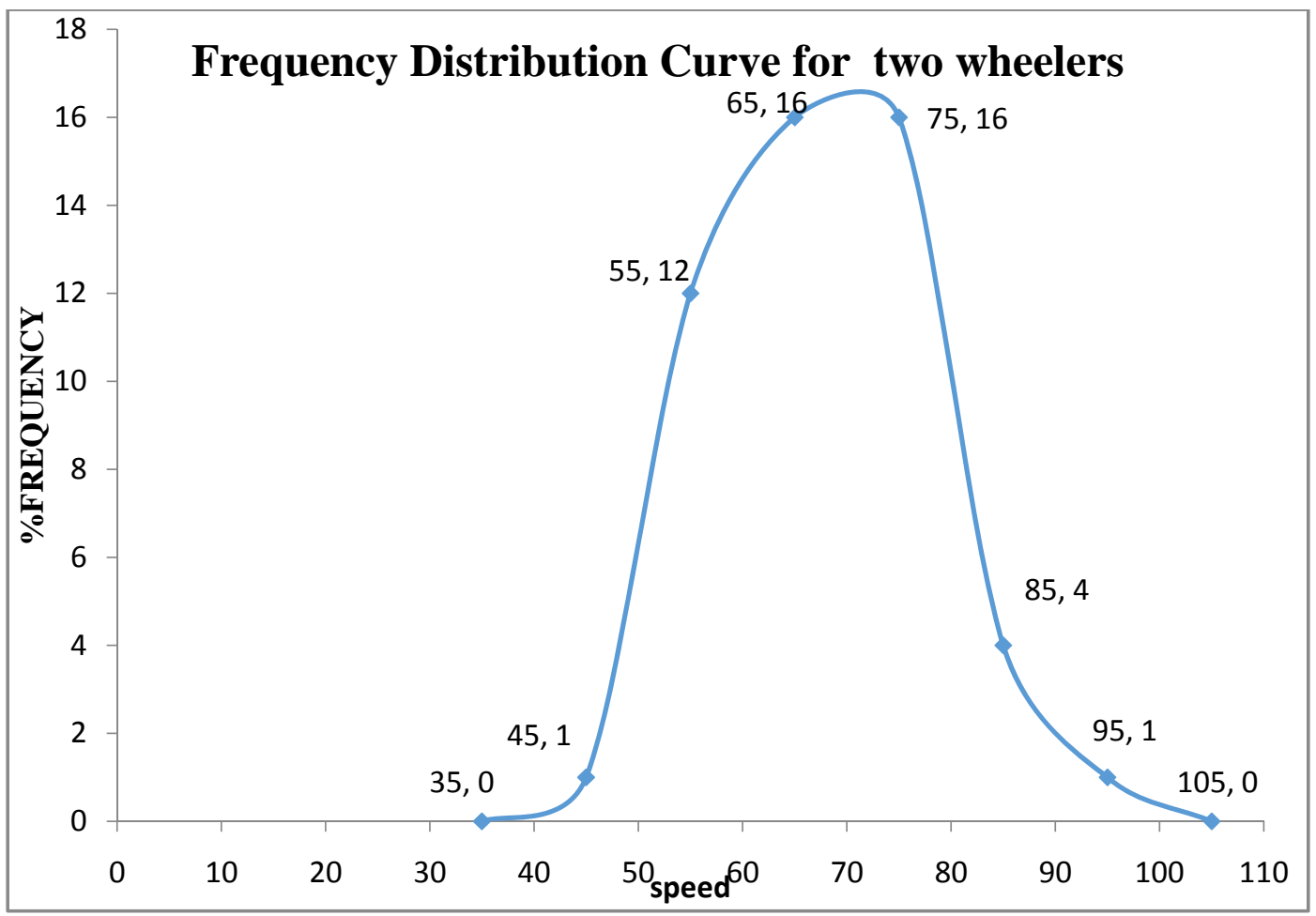

Fig. 8 Frequency Distribution Curve of Two Wheelers

From frequencyDistribution curveModal speed $=65 \mathrm{kmph}$

Therefore the most preferred speed at which maximumProportion of trucks travel is the MODAL SPEED $=$ $65 \mathrm{KMPH}$

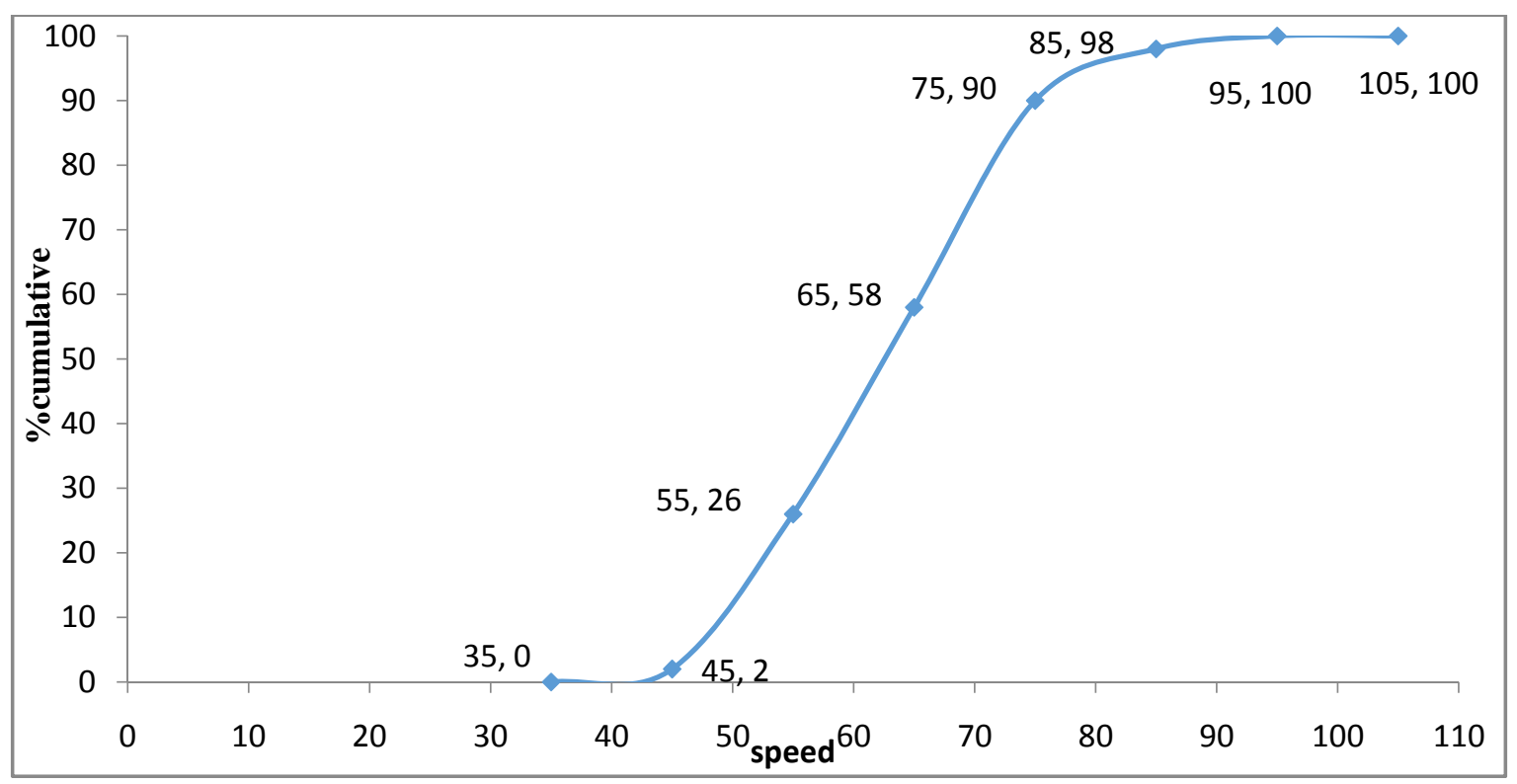

Fig. 9 Cumulative Frequency Distribution Curve for Two Wheelers

FOR NH1 CHAHERU FOR TWO WHEELERS

1. UPPER SPEED $=85^{\text {th }}$ percentile speed $=70 \mathrm{kmph}$

2. LOWER SPEED $=15^{\text {th }}$ percentile speed $=45 \mathrm{kmph}$

3. DESIGN SPEED $=98^{\text {th }}$ percentile speed $=85 \mathrm{kmph}$

4. MEDIAN SPEED $=50^{\text {th }}$ percentile speed $=60 \mathrm{kmph}$ 
TABLE 8. Data Analysis of Two Wheelers

\begin{tabular}{|c|c|c|c|c|}
\hline SPEED RANGE Kmph & MID SPEED (v) & $\mathbf{( q )}$ & $\mathbf{( q . v . )}$ & $\mathbf{( q / v )}$ \\
\hline $20-30$ & 25 & 0 & 0 & 0.0 \\
\hline $30-40$ & 35 & 0 & 0 & 0.0 \\
\hline $40-50$ & 45 & 1 & 45 & 0.0222 \\
\hline $50-60$ & 55 & 12 & 660 & 0.2181 \\
\hline $60-70$ & 65 & 16 & 1040 & 0.2461 \\
\hline $70-80$ & 75 & 16 & 1200 & 0.2133 \\
\hline $80-90$ & 85 & 4 & 340 & 0.0470 \\
\hline $90-100$ & 95 & 1 & 95 & 0.0105 \\
\hline & & $\sum \mathrm{f}=50$ & $\sum \mathrm{qv}=3380$ & $\sum \mathrm{q} / \mathrm{v}=0.7572$ \\
\hline
\end{tabular}

Space mean speed (Vs)

Time mean speed $(\mathrm{Vt})$

$=\sum \frac{q}{v} / \sum f=(0.7572) /(50)=0.01514 \mathrm{kmph}$

Standard deviation (S2)

$=\sum q v / \sum f=(3380) / 50=67.6 \mathrm{kmph}$

$=\mathrm{Vs}(\mathrm{Vt}-\mathrm{Vs})=0.01514(67.6-0.01514)=1.023$

TABLE 9. Frequency Distribution of Two Wheelers Maheru Road

\begin{tabular}{|c|c|c|c|c|c|}
\hline \multicolumn{3}{|c|}{$\begin{array}{l}\text { LOCATION: CHAHERU } \\
\text { WEATHER: SUNNY DAY } \\
\text { TYPE OF ROAD : MAHERU ROAD } \\
\text { MEASUREMENT TECHNIQUE: MANUALLY }\end{array}$} & \multicolumn{3}{|c|}{$\begin{array}{l}\text { DATE: } 2 / 10 / 16 \\
\text { TIME: } 10 \text { A.M. TO 2P.M. } \\
\text { ASE LENGTH: 50m } \\
\text { VEHICLE: Two wheelers (50) }\end{array}$} \\
\hline $\begin{array}{c}\text { SPEED } \\
\text { RANGE Kmph }\end{array}$ & $\begin{array}{c}\text { MID } \\
\operatorname{SPEED}(x)\end{array}$ & $\begin{array}{l}\text { FREQUENCY } \\
\text { (f) }\end{array}$ & $\begin{array}{c}\text { \%FREQUENCY } \\
\text { (\%f) }\end{array}$ & $\begin{array}{l}\text { \%CUMMULATIVE } \\
\text { FREQUENCY } \\
\text { (\%c.f) }\end{array}$ & (fx) \\
\hline $10-20$ & 15 & 0 & 0 & 0 & 0 \\
\hline $20-30$ & 25 & 15 & 30 & 30 & 375 \\
\hline $30-40$ & 35 & 26 & 52 & 82 & 910 \\
\hline $40-50$ & 45 & 6 & 12 & 94 & 270 \\
\hline $50-60$ & 55 & 2 & 4 & 98 & 110 \\
\hline \multirow[t]{2}{*}{$60-70$} & 65 & 1 & 2 & 100 & 65 \\
\hline & & $\sum \mathrm{f}=50$ & & & $\sum \mathrm{fx}=1730$ \\
\hline
\end{tabular}

AVERAGE SPEED FOR TWO WHEELER $=\sum f x / \sum f=(1730 / 50)=34.6 \mathrm{kmph}$ 


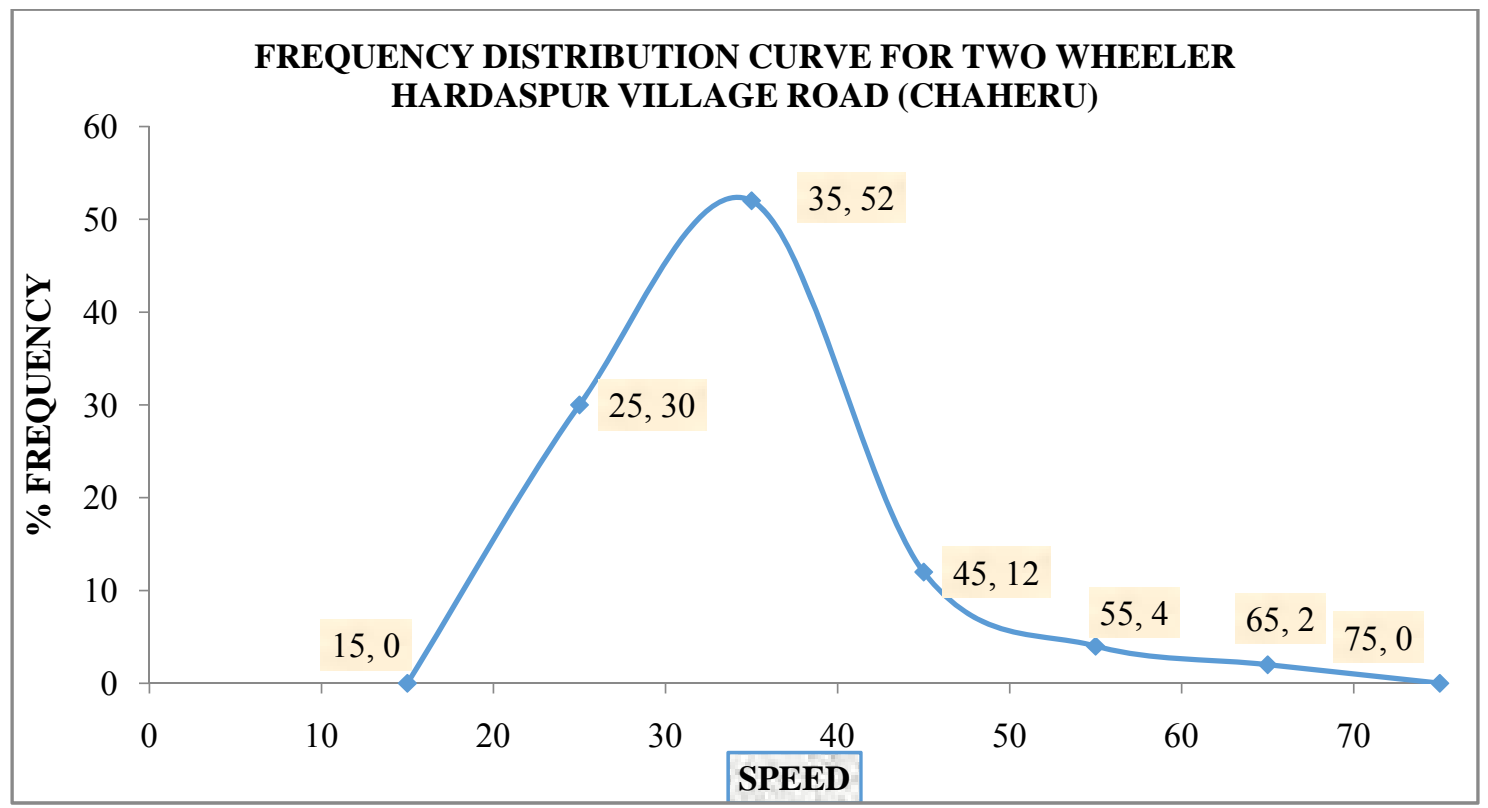

Fig. 10 Frequency Distribution Curve of Two Wheelers Maheru Road

From frequencyDistribution curveModal speed $=52 \mathrm{kmph}$

Therefore the most preferred speed at which maximumProportion of trucks travel is the MODAL SPEED $=52$ $\mathrm{KMPH}$

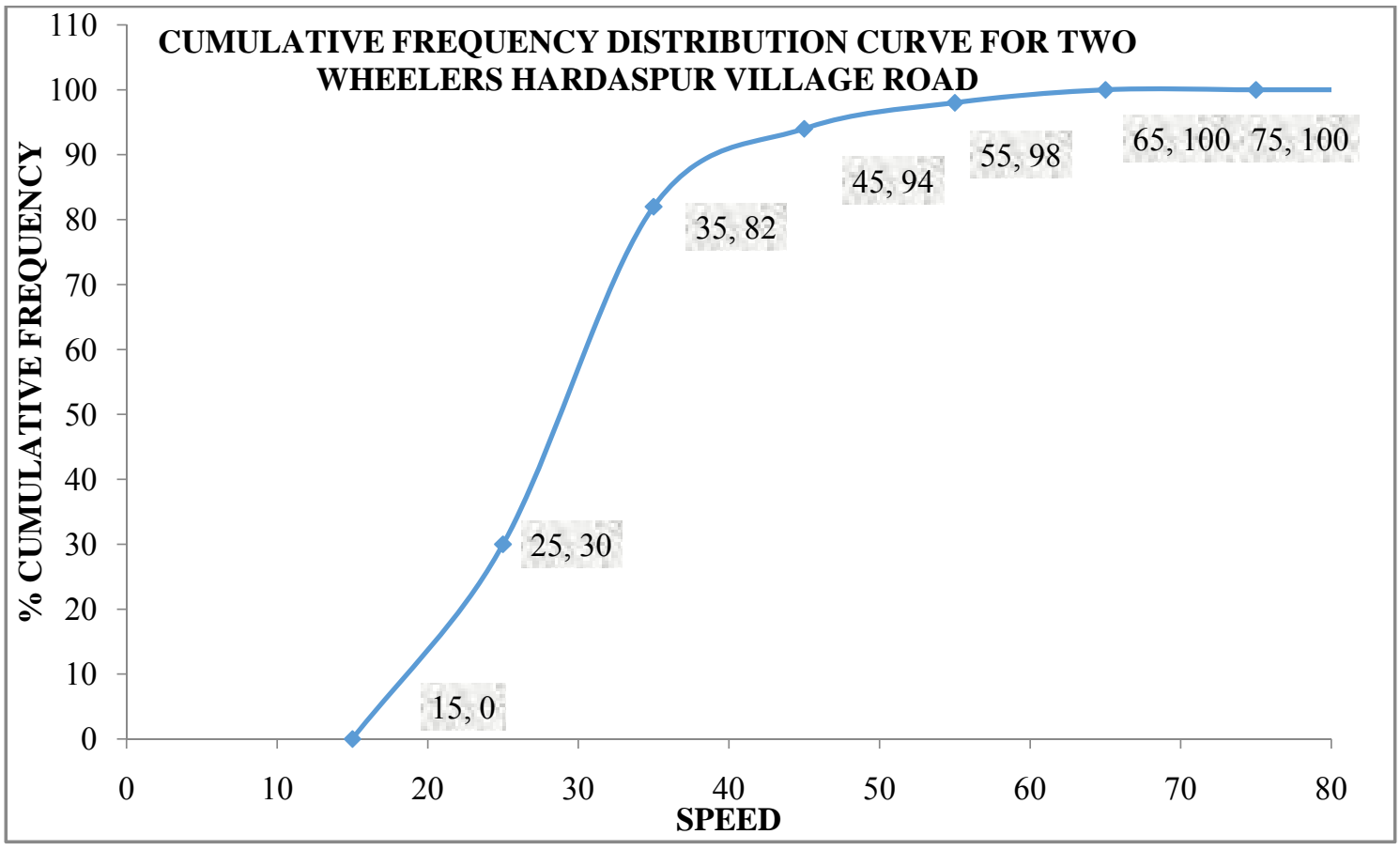

Fig. 11 Cumulative Frequency Distribution Curve for Two Wheelers Maheru Road

FOR VR CHAHERU FOR TWO WHEELERS
1. UPPER SPEED
2. LOWER SPEED
$=\quad 85^{\text {th }}$ percentile speed $=40 \mathrm{kmph}$
3. DESIGN SPEED
$15^{\text {th }}$ percentilespeed $=20 \mathrm{kmph}$
4. MEDIAN SPEED
$98^{\text {th }}$ percentile speed $=55 \mathrm{kmph}$
$=\quad 50^{\text {th }}$ percentile speed $=30 \mathrm{kmph}$


TABLE 10. Data Analysis of Two Wheelers Maheru Road

\begin{tabular}{|c|c|c|c|c|}
\hline SPEED RANGE Kmph & MID SPEED (v) & (q) & (q.v.) & (q/v) \\
\hline $10-20$ & 15 & 0 & 0 & 0.000 \\
\hline $20-30$ & 25 & 15 & 375 & 0.600 \\
\hline $30-40$ & 35 & 26 & 910 & 0.7428 \\
\hline $40-50$ & 45 & 6 & 270 & 0.1333 \\
\hline $50-60$ & 55 & 2 & 110 & 0.03636 \\
\hline $60-70$ & 65 & 1 & 65 & 0.01538 \\
\hline & & $\sum \mathrm{f}=50$ & $\sum \mathrm{qv}=1730$ & $\sum \mathrm{q} / \mathrm{v}=1.90682$ \\
\hline
\end{tabular}

Space mean speed $(\mathrm{Vs})=\sum \frac{q}{v} / \sum f=(1.9068) /(50)=0.0381 \mathrm{kmph}$

Time mean speed $(\mathrm{Vt}) \quad=\sum q v / \sum f=(1730) / 50=34.6 \mathrm{kmph}$

Standard deviation $(\mathrm{S} 2)=$ Vs. $(\mathrm{Vt}-\mathrm{Vs})=0.0381(34.6-0.0381)=1.31$

A. For NH-1 Chaheru

\section{CONCLUSION}

1.) Modal Speed:

$\begin{array}{lll}\text { BUS } & =65 \mathrm{kmph} \\ \text { CAR } & =55 \mathrm{kmph} \\ \text { TRUCK } & \\ \text { TWO WHEELER } & =70 \mathrm{kmph}\end{array}$

B. For Village Road Chaheru
1.) Modal Speed:
TWO WHEELER
$=35 \mathrm{kmph}$

SPEED TO CHECK DESIGN ELEMENT $=98^{\mathrm{TH}}$ PERCENTILE SPEED

Speed is one of the development operational parts that should be considered in the layout of the road geometrics. The safe acceptable speed most remote indicate will make vehicles go in a sorted out and safe way. The effects of road geometrical design on the speed of the vehicles exhibit that the differences in road geometrical framework would particularly impact the speed of the vehicles. The design speed should be made depending upon the shielded speed limit of the road. The vehicles found in the lower 15 percent are considered to be travelling unreasonably direct and those saw over the 85th percentile are thought to outperform a secured and reasonable speed. 85th percentile could be a govern in setting up the speed limit as this speed is seen as protected and reasonable under conditions states of the road.

\section{ACKNOWLEDGEMENT}

I would like to express my gratitude to all the people behind the screen who helped me to transform an idea into a real application. I would like to thank my student Jyothi Aind for her technical assistance, and support in carrying out my work at college. The satisfaction and euphoria that accompany the successful completion of the task would be great but incomplete without the mention of the people who made it possible with their constant support and encouragement crowns all the efforts with success. In this context I would like to thank all the other staff members, both teaching and non-teaching, who have extended their timely help and eased my task.

In addition, I would like to thank all the professors of the department of civil engineering for all the knowledge they have shared with me.

Also, I would like to express my love and gratitude to my beloved parents for their understanding and endless love throughout my journey.

\section{REFERENCES}

[1] Agent K.R Pigman. Evaluation of Speed Limits in Kentucky. Journal of Transportation Research Records. 1998; VI(1541): p. 57-64.

[2] Dharamveer Singh MZ. Modelling of 85th Percentile Speed for Rural Highways for Enhanced Traffic Safety. 9th ed. Justo K\&, editor. Roorkee: Nem Chand \& Bros; 2008.

[3] Fitzpatrick K, Carlson, P., Brewer, M.A., Wooldridge, M.D., and Miaou, S.P... Design Speed, Operating Speed, and Posted Speed Practices. NCHRP, Department of Transportation Engineering; 2003. Report No.: 504.

[4] L.R K. Traffic Engineering \& Transport Planning. 7th ed. New Delhi: Khanna Publications; 2001.

[5] M.Zainora Asma LHMTp. Planning Malaysia: Urban Planning and Local Governance. Transportation Research Records. 2014; III(567): p. 15-26.

[6] D S. Accidents on Main Rural Highways Related to Speed, Driver, \& Vehicle. Kansas: Bureau of Public Roads, Department of Commerce; 1964. 